\title{
AUTOMATIC CARDIAC FLOW QUANTIFICATION ON 3D VOLUME COLOR DOPPLER DATA
}

\author{
Yang Wang ${ }^{1}$, Bogdan Georgescu ${ }^{1}$, Saurabh Datta ${ }^{2}$, Shizhen Liu ${ }^{3}$, Mani A. Vannan ${ }^{3}$, Dorin Comaniciu ${ }^{1}$ \\ ${ }^{1}$ Siemens Corporate Research, Princeton, NJ $\quad{ }^{2}$ Siemens Ultrasound, Mountain View, CA \\ ${ }^{3}$ The Ohio State University Medical Center, Columbus, $\mathrm{OH}$
}

\begin{abstract}
Valvular heart diseases are recognized as a significant cause of morbidity and mortality. Accurate quantification of cardiac flow volumes in patients is essential in evaluation of the progression of the disease and in determination of clinical options. Recent advances in the real-time 3D full volume echocardiography have enabled high frame rate acquisition of volumetric color Doppler flow images. In this paper, we propose a fully automated method to quantify the cardiac flow using instantaneous $3 \mathrm{D}+\mathrm{t}$ ultrasound data. The anatomical information such as mitral annulus and left ventricle outflow tract (LVOT) are detected and tracked automatically accounting for the heart motion. Furthermore, the proposed method automatically detects and tracks the endocardial boundary of the left ventricle (LV) and computes the instantaneous change in LV volume. This information is used to overcome inherent limitation of the color Doppler velocity ambiguity such that de-aliasing parameters are computed and used to correct flow computations. Preliminary results with clinical data presented here agree well with accepted clinical measurements in a quantitative manner. The proposed method is efficient and achieves high speed performance of 0.2 second per volume of ultrasound data.
\end{abstract}

Index Terms - Color Doppler, Anatomy Detection, Motion Tracking, Learning-Based Methods

\section{INTRODUCTION}

The quantification of flow volume is important for evaluation of patients with cardiac dysfunction and cardiovascular disease. However, the accurate flow quantification remains a significant challenge for cardiologists [1]. Doppler ultrasound is a non-invasive and cost effective method for evaluation of intracardiac blood flow, for assessment of cardiac function, for estimation of shunt flows in congenital cardiac defects, and for assessment regurgitation in the presence of valvular disease. With real-time full volume echocardiography it is now feasible to acquire transthoracic 3D color flow imaging (CFI) for every heartbeat (without stitching) such that both mitral valve and LVOT can be covered by color Doppler region of interest. However, a fundamental limitation of flow velocity aliasing remains which can introduce significant errors in flow quantification directly using color Doppler data. The velocity ambiguity cannot be overcome just by ultrasound data especially when true velocity is several multiples of the Nyquist level [2]. To address this issue, various approaches have been proposed using customized hardware [3] or certain geometric assumptions $[4,5,6,7]$.

In this paper, we propose a fully automated method for cardiac flow volume quantification using instantaneous $3 \mathrm{D}+\mathrm{t}$ ultrasound data. More specifically, our method automatically detects both the mitral annulus and left ventricular outflow tract (LVOT) and places measurement planes at appropriate locations. To compensate non-rigid heart motion, measurement planes are tracked through the whole cardiac cycle to adjust the sampling locations and orientations in each frame. As a result, the flow volumes are computed consistently based on the anatomical structure of the left ventricle (LV). Furthermore, to recover the de-aliasing factor of the color Doppler flow data, our method also estimates the LV volume change by automatically detecting and tracking the endocardial boundary on the ultrasound data. A de-aliasing approach is proposed to compute the volume of both mitral inflow and LVOT outflow. To demonstrate the performance, we evaluated our method on a clinical dataset taken from 22 normal subjects. The comparison of measurements with the proposed method with two routine clinical measurements provide a quantitative comparison with existing reference methods. The clinical measurements used for comparison are 2D quantitative Doppler with pulsed wave Doppler acquisition at LVOT to estimate LV stroke volume and LV stroke volume from 3D b-mode contouring of LV cavity. These are clinically accepted methods and independently validated elsewhere.

\section{FRAMEWORK}

In this section, we present the new framework to estimate 3D volume of both the mitral and LVOT flow. As illustrated in Figure 1, our system includes the following main steps:

1. Automatic anatomy detection: In the first frame, the endocardial boundary of the left ventricle (LV), the mitral annulus, and the left ventricular outflow tract (LVOT) are detected using Marginal Space Learning (MSL) [8]. 

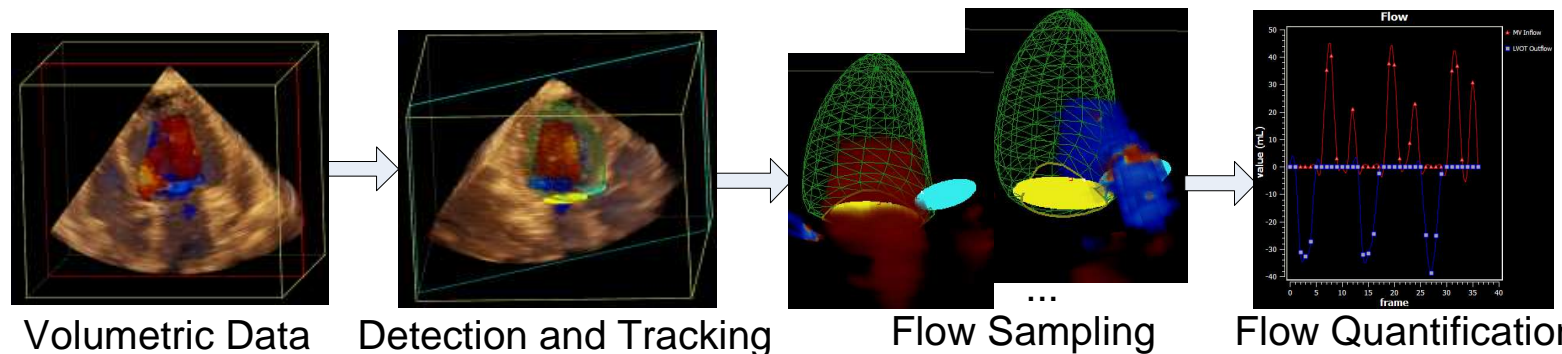

Fig. 1. The outline of our automatic flow volume quantification framework.

2. Cardiac motion tracking: The 3D motion of the left ventricle, including the ventricular wall, the mitral annulus, and the LVOT, are estimated by fusing information from multiple cues, including optical flow, boundary detection, and motion prior.

3. Three-dimensional flow sampling: The tracked locations of the mitral annulus and LVOT are used to construct and adjust the sampling planes of the color flow data. Consequently, the flow volume is computed by aggregating the sampled color flow values in the threedimensional space.

4. Flow de-aliasing: Based on the tracked LV endocardial boundaries, the LV volume change is computed between neighboring frames to estimate the de-aliasing factor of the measured color Doppler data. The dealiased flow volumes are computed for both the mitral inflow and the LVOT outflow.

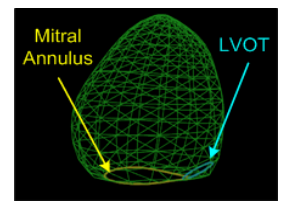

(a)

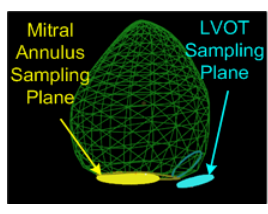

(b)

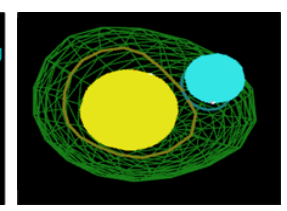

(c)
Fig. 2. The anatomical model used to represent the left ventricle, mitral annulus, and left ventricular outflow tract (LVOT). (a) is the side and bottom views of the 3D mesh model, where the mitral annulus and LVOT are highlighted in the yellow and blue color, respectively. (b) and (c) are the side and bottom views of the sampling planes at the mitral annulus and LVOT, respectively.

\subsection{Anatomical Model}

To represent the anatomical structure of the left ventricle, mitral annulus, and left ventricular outflow tract, a 3D mesh model is used in our system as illustrated in Figure 2(a-b). Figure 2(a) shows the side view of our 3D model with the mitral annulus and the LVOT ring highlighted in the yellow and blue color, respectively. The sampling planes at the mitral annulus and LVOT are illustrated in Figure 2(b) and (c). Because of the physical form of the 3D ultrasound pyramid, a plane in the acoustic space with a constant distance to the transducer corresponds to a Gaussian sphere in the Cartesian space, centering at the tip of the pyramid. Therefore, the sampling plane in our model is defined on a Gaussian sphere passing through the mitral annulus or LVOT. To compute the integral volume of the mitral inflow and LVOT outflow, we consider the circular area enclosed by the mitral annulus and LVOT ring, as shown in Figure 2(c).

\subsection{Learning-based anatomy detection}

In the starting frame (typically the end-diastole cardiac phase), we detect automatically the endocardial boundary of the left ventricle (LV), the mitral annulus, and the left ventricular outflow tract (LVOT). A 3D detector is learned to locate the pose, including the position $X=(x, y, z)$, orientation $\theta=(\alpha, \beta, \gamma)$ and scale $S=\left(s_{x}, s_{y}, s_{z}\right)$, of the LV using the marginal space learning (MSL) approach [8]. The local deformations of the mitral annulus, LVOT, and myocardial boundaries are further estimated based on the posterior distribution $p_{i}(X \mid I)$ of each control point on the surface, which is learned using the steerable features and the probability boosting-tree (PBT) [9].

\subsection{Cardiac motion tracking}

Starting from the detection result at the initial frame, the model deformations are propagated to neighboring frames using both the learned features and the local image templates. To ensure temporal consistency and smooth motion and to avoid drifting and outliers, two collaborative trackers, an optical flow tracker and a boundary detection tracker, are used in our method. The optical flow tracker directly computes the temporal displacement for each point from one frame to the next, while the detection tracker obtains the deformations in each frame with maximal probability. The above two trackers are integrated into a single Bayesian framework, with the assumption that the input images $I$ are mutually independent [10]:

$$
\arg \max _{\vec{X}_{t}} p\left(\vec{X}_{t} \mid \vec{Y}_{1: t}\right)=\arg \max _{\vec{X}_{t}} p\left(\vec{Y}_{t} \mid \vec{X}_{t}\right) p\left(\vec{X}_{t} \mid \vec{Y}_{1: t-1}\right),
$$

where $\vec{Y}_{1: t}=\left(\vec{Y}_{1}, \ldots, \vec{Y}_{t}\right)$ are the measurements from the first $t$ frames $I_{1: t}=\left(I_{1}, \ldots, I_{t}\right)$. For clarity, we use $\vec{X}_{t}$ to denote a concatenation of the mesh point positions, $\vec{X}_{t}=$ $\left[X_{1}, \cdots, X_{n}\right]$, which need to be estimated at the current time instance $t$, and $n$ is the total number of points in the model.

The likelihood term $p\left(\vec{Y}_{t} \mid \vec{X}_{t}\right)$ is computed from both boundary detection and local image template matching, i.e.,

$$
p\left(\vec{Y}_{t} \mid \vec{X}_{t}\right)=(1-\lambda) p\left(F_{t} \mid \vec{X}_{t}\right)+\lambda p\left(T_{t} \mid \vec{X}_{t}\right),
$$


where $F_{t}$ is the steerable feature response [8], $T_{t}$ is the local image template, and $\lambda$ is the weighting coefficient of the matching term. Given the resulting shapes $\vec{X}_{1: t-1}$ from the previous $t-1$ frames, the prediction term $p\left(\vec{X}_{t} \mid \vec{Y}_{1: t-1}\right)$ can be simplified as $p\left(\vec{X}_{t} \mid \vec{X}_{1: t-1}\right)$, which can be learned from the training data set as in [11]. The motion prior is estimated at the training stage using motion manifold learning and hierarchical K-means clustering, from a pre-annotated database of sequences containing one cardiac cycle each. Firstly the temporal deformations are aligned by $4 \mathrm{D}$ generalized procrustes analysis. Next a low-dimensional embedding is computed from the aligned training sequences using the ISOMAP algorithm [12]. Finally, in order to extract the modes of motion, the motion sequences are then clustered with hierarchical Kmeans based on the Euclidean distance in the lower dimensional manifold.

The above deformation propagation step is repeated until the full 4D model is estimated for the complete sequence. In this way the collaborative trackers complement each other, as the optical flow tracker provides temporally consistent results and its major issue of drifting is addressed by the boundary detection. Finally to obtain a smooth motion field, the tracking is performed in both forward and backward directions given the periodic nature of the cardiac motion.

\subsection{Flow computation and de-aliasing}

Given the tracking result $\vec{X}$ from Section 2.3, the two planes as shown in Figure 2(b,c) are constructed to sample and compute the mitral and LVOT flow. Figure 3 shows the examples of flow sampling on two different frames, one with the mitral inflow and the other with LVOT outflow. The mitral annulus and LVOT sampling planes are highlighted in the yellow and blue color, respectively. Thus, given a color flow image $F_{t}$ at the time instance $t$, the flow volume is computed as an integral of the color measurements on the sampling plane:

$$
\begin{aligned}
V F_{t}^{m a} & =d A \times V F_{t}^{m} / 128 / f r \times v s \\
V F_{t}^{m} & =\sum_{i=1}^{N_{s}} F_{t}(X \overrightarrow{(i)})
\end{aligned}
$$

where $d A$ is the unit sampling area on the sampling plane, $f r$ is the frame rate, $v s$ is the Doppler velocity scale, $N_{s}$ is the number of non-zero samples on the sample plane, and $X \overrightarrow{(i})$ is the $3 \mathrm{D}$ position of the $i-t h$ sampling area.

In color flow images, aliasing is a common issue which describes single or multiple exceeding of the color Doppler Nyquist velocity, causing ambiguity for velocities beyond the Nyquist level [2]. In our proposed method, the LV volume $V_{t}$ can be computed based on the tracking result $\vec{X}$ from Section 2.3 for each frame $t$. As a result, the LV volume change can be computed as the difference between two neighboring frames as follows:

$$
d V_{t}=V_{t}-V_{t-1}
$$

Since the LV volume change $d V$ and the flow volume $V F_{m a}$ measures the same amount of blood flow through the left ven-

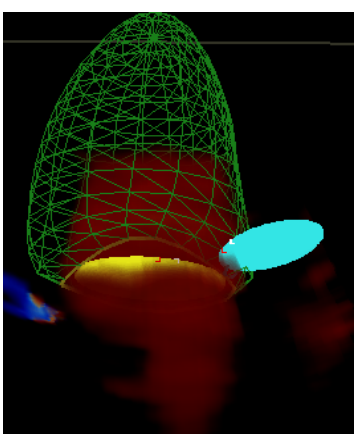

(a) Mitral inflow

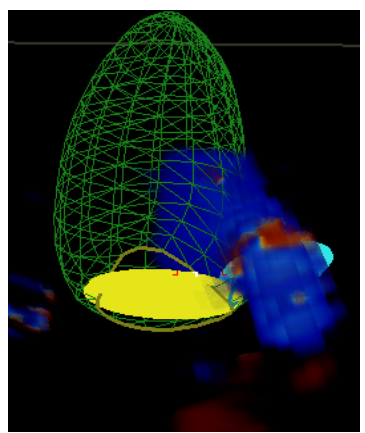

(b) LVOT outflow
Fig. 3. Flow sampling examples. (a) and (b) show the flow sampling on two different frames. The mitral annulus and LVOT sampling planes are highlighted in the yellow and blue color, respectively.

tricle at certain time instance, the de-aliasing factor of the color flow measurement can be computed as the ratio between two volume values, i.e.,

$$
f_{d e}=\left\lfloor\frac{d V_{t}-V F_{t}^{m a}}{d A \times N_{s} / f r \times v s}\right\rfloor
$$

where $d A$ is the unit sampling area, $N_{s}$ is the number of nonzero samples on the sample plane, $f r$ is the frame rate, and $v s$ is the Doppler velocity scale, and $\lfloor x\rfloor$ is the floor function which returns the closest integer not greater than $x$.

\section{EXPERIMENTAL RESULTS}

Figure 4 shows a multi-beat example from a normal patient. Figure 4(a) shows the aliased flow measurement sampled from the color flow images, while Figure 4(b) shows the resulting flow from our de-aliasing method. The input sequence has 37 volumes with 3 heart beats.

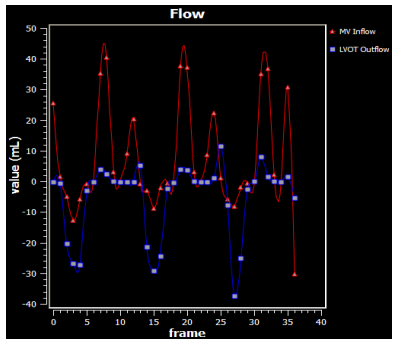

(a) Aliased flow

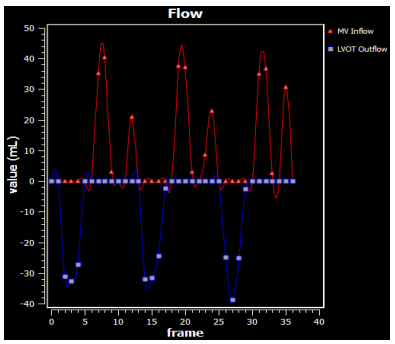

(b) De-aliased flow
Fig. 4. Example flow estimation on a normal case. (a) shows the original flow volume measurement from color flow images with velocity aliasing. (b) is the resulting flow volume from our de-aliasing method. The mitral inflow is plotted in the red curve while the LVOT outflow is in blue. The de-aliased mitral inflow and LVOT outflow volume curves show a consistent pattern in all three cycles.

To evaluate the performance of our method, a set of 3D full-volume ultrasound sequences were acquired by a Siemens SC2000 scanner with an average volume rate of 15 vps at the Ohio State University Medical Center. 22 subjects with normal valves were enrolled with the IRB approval. 
Figures 5 and 6 report the comparison between the expert measurements using 2D pulsed wave (PW) Doppler and the flow volumes estimated by our method. The LV stroke volume (LVSV) was very close to the volume from LVOT-PW $(70.1 \pm 20.8 \mathrm{ml}, 69.7 \pm 16.7 \mathrm{ml})$ with good correlation $(r=0.78)$. 3-D LV inflow and outflow volumes $(73.6 \pm 16.3$ $\mathrm{ml}, 67.6 \pm 14.6 \mathrm{ml})$ were correlated well with LVSV and LVOT-PW respectively $(r=0.77,0.91)$. Therefore all the estimated flow volumes were consistent and close to the expert measurements, which demonstrated the accuracy and robustness of our proposed method.

\begin{tabular}{|r|c|c|c|}
\hline Measure (ml) & Mean & STD & Std. Error Mean \\
\hline \hline LVOT-PW & 69.7 & 16.7 & 3.6 \\
\hline LVSV & 70.1 & 20.8 & 4.4 \\
\hline 3D CD Mitral Inflow & 73.6 & 16.3 & 3.5 \\
\hline 3D CD LVOT Outflow & 67.6 & 14.6 & 3.1 \\
\hline
\end{tabular}

Fig. 5. Flow measure comparison on 22 normal patients. The first row shows the LVOT outflow volume measured by a clinical expert using 2D pulsed wave (PW) Doppler. The second row is the estimated LV stroke volume using the delineated LV endocardial boundary on the volumetric b-mode ultrasound data. The last two rows are the de-aliased mitral inflow and LVOT outflow based on the sampled volumetric color Doppler data by our method. The estimated flow volumes are consistent between all four measurements and close to the expert measurements, which demonstrates the accuracy and robustness of our proposed method.

\begin{tabular}{|r|c|c|c|}
\hline Measure 1 & Measure 2 & correlation & p-value \\
\hline \hline LVOT-PW & LVSV & 0.78 & $<0.001$ \\
\hline 3D CD Mitral Inflow & LVSV & 0.77 & $<0.001$ \\
\hline 3D CD LVOT Outflow & LVOT-PW & 0.91 & $<0.001$ \\
\hline
\end{tabular}

Fig. 6. Correlation and statistical significance testing of flow measure on 22 normal patients between (1) the LVOT outflow volume measured using 2D pulsed wave (PW) Doppler and the estimated LV stroke volume; (2) the LVOT and the de-aliased Mitral inflow by our method; and (3) the LVOT-PW and the LVOT outflow by our method. The comparison shows good correlation between all measurements which is statistically significant.

\section{CONCLUSION}

In this paper, we present a fully automatic method to estimate both mitral inflow and LVOT outflow on 3D real-time full volume ultrasound data. A 3D model is fitted automatically to the left ventricle (LV), mitral annulus, and LVOT to construct measurement planes in a volumetric color Doppler image. To compensate heart motion, multiple information sources, such as image gradients, boundary detection and motion prediction, are fused to achieve a robust tracking through the whole cardiac cycle. Furthermore, given the tracked LV endocardial boundaries, a new approach is proposed to correct for aliasing in the color Doppler data by using LV volume change between two neighboring frames. Preliminary results on clinical data showed good correlation with expert measurements in two reference methods. The proposed method is efficient and achieves high speed performance of 0.2 second per frame for volumetric ultrasound data.

\section{REFERENCES}

[1] Little, S.H.: Quantifying mitral valve regurgitation: New solutions from the 3 rd dimension. Journal of the American Society of Echocardiography 23(1) (2010) $9-12$

[2] Hatle, L., Angelsen, B.: Doppler ultrasound in cardiology: physical principles and clinical applications. Lea \& Febinger

[3] Skaug, T.R., Hergum, T., Amundsen, B.H., Skjrpe, T., Torp, H., Haugen, B.O.: Quantification of mitral regurgitation using high pulse repetition frequency three-dimensional color doppler. Journal of the American Society of Echocardiography 23(1) (2010) $1-8$

[4] Zoghbi, W.A., Enriquez-Sarano, M., Foster, E., Grayburn, P.A., Kraft, C.D., Levine, R.A., Nihoyannopoulos, P., Otto, C.M., Quinones, M.A., Rakowski, H., Stewart, W.J., Waggoner, A., Weissman, N.J.: Recommendations for evaluation of the severity of native valvular regurgitation with twodimensional and doppler echocardiography. Journal of the American Society of Echocardiography 16(7) (2003) 777 - 802

[5] Matsumura, Y., Fukuda, S., Tran, H., Greenberg, N.L., Agler, D.A., Wada, N., Toyono, M., Thomas, J.D., Shiota, T.: Geometry of the proximal isovelocity surface area in mitral regurgitation by 3-dimensional color doppler echocardiography: Difference between functional mitral regurgitation and prolapse regurgitation. American Heart Journal 155(2) (2008)

[6] Yosefy, C., Levine, R.A., Solis, J., Vaturi, M., Handschumacher, M.D., Hung, J.: Proximal flow convergence region as assessed by real-time 3-dimensional echocardiography: Challenging the hemispheric assumption. Journal of the American Society of Echocardiography 20(4) (2007) 389-396

[7] Plicht, B., Kahlert, P., Goldwasser, R., Janosi, R.A., Hunold, P., Erbel, R., Buck, T.: Direct quantification of mitral regurgitant flow volume by real-time three-dimensional echocardiography using dealiasing of color doppler flow at the vena contracta. Journal of the American Society of Echocardiography 21(12) (2008) $1337-1346$

[8] Zheng, Y., Barbu, A., Georgescu, B., Scheuering, M., Comaniciu, D.: Four-chamber heart modeling and automatic segmentation for 3-D cardiac CT volumes using marginal space learning and steerable features. TMI 27(11) (2008) 1668-1681

[9] Tu, Z.: Probabilistic boosting-tree: Learning discriminative models for classification, recognition, and clustering. In: ICCV. (2005) II: 1589-1596

[10] Zhu, Y., Papademetris, X., Sinusas, A.J., Duncan, J.S.: A dynamical shape prior for lv segmentation from RT3D echocardiography. In: Proc. Int'l Conf. Medical Image Computing and Computer Assisted Intervention. (2009) 206-213

[11] Wang, Y., Georgescu, B., Comaniciu, D., Houle, H.: Learningbased 3D myocardial motion flow estimation using high frame rate volumetric ultrasound data. In: ISBI. (2010) 1097-1100

[12] Tenenbaum, J.B., de Silva, V., Langford, J.C.: A global geometric framework for nonlinear dimensionality reduction. Science 290(5500) (2000) 2319- 2323 